\title{
Engineering Escherichia coli for high-yield geraniol production with biotransformation of geranyl acetate to geraniol under fed-batch culture
}

Wei Liu' ${ }^{1,2,3}$, Xin Xu ${ }^{1,3}$, Rubing Zhang ${ }^{1,3}$, Tao Cheng ${ }^{1}$, Yujin Cao ${ }^{1}$, Xiaoxiao Li $^{1}$, Jiantao Guo ${ }^{4}$, Huizhou Liu* and Mo Xian ${ }^{1 *}$

\begin{abstract}
Background: Geraniol is an acyclic monoterpene alcohol, which exhibits good prospect as a gasoline alternative. Geraniol is naturally encountered in plants at low concentrations and an attractive target for microbial engineering. Geraniol has been heterologously produced in Escherichia coli, but the low titer hinders its industrial applications. Moreover, bioconversion of geraniol by E. coli remains largely unknown.

Results: Recombinant overexpression of Ocimum basilicum geraniol synthase, Abies grandis geranyl diphosphate synthase, and a heterotic mevalonate pathway in E. coli BL21 (DE3) enabled the production of up to $68.6 \pm 3 \mathrm{mg} / \mathrm{L}$ geraniol in shake flasks. Initial fed-batch fermentation only increased geraniol production to $78.8 \mathrm{mg} / \mathrm{L}$. To further improve the production yield, the fermentation conditions were optimized. Firstly, $81.4 \%$ of volatile geraniol was lost during the first $5 \mathrm{~h}$ of fermentation in a solvent-free system. Hence, isopropyl myristate was added to the culture medium to form an aqueous-organic two-phase culture system, which effectively prevented volatilization of geraniol. Secondly, most of geraniol was eventually biotransformed into geranyl acetate by E. coli, thus decreasing geraniol production. For the first time, we revealed the role of acetylesterase (Aes, EC 3.1.1.6) from E. coli in hydrolyzing geranyl acetate to geraniol, and production of geraniol was successfully increased to $2.0 \mathrm{~g} / \mathrm{L}$ under controlled fermentation conditions.

Conclusions: An efficient geraniol production platform was established by overexpressing several key pathway proteins in engineered $E$. coli strain combined with a controlled fermentation system. About $2.0 \mathrm{~g} / \mathrm{L}$ geraniol was obtained using our controllable aqueous-organic two-phase fermentation system, which is the highest yield to date. In addition, the interconversion between geraniol and geranyl acetate by E. coli was first elucidated. This study provided a new and promising strategy for geraniol biosynthesis, which laid a basis for large-scale industrial application.
\end{abstract}

Keywords: Geraniol, Geranyl acetate, Fed-batch fermentation, Acetylesterase, Engineered Escherichia coli

\section{Background}

Monoterpene geraniol (trans-isomer of 3,7-dimethyl-2, 6-octadiene-1-ol), which is emitted from flowers, has been widely applied in perfume, pharmaceutical, and other industries [1-3]. As a gasoline alternative, geraniol

\footnotetext{
*Correspondence: liuhuizhou@qibebt.ac.cn; xianmo1@qibebt.ac.cn

${ }^{1}$ CAS Key Laboratory of Bio-Based Materials, Qingdao Institute

of Bioenergy and Bioprocess Technology, Chinese Academy of Sciences,

Qingdao 266101, China

Full list of author information is available at the end of the article
}

is superior to ethanol due to low hygroscopicity, high energy content, and relatively low volatility $[4,5]$. Geraniol is derived from geranyl diphosphate (GPP) which is synthesized from either the mevalonate (MVA) pathway or the methylerythritol phosphate pathway in plants [6, 7]. However, geraniol has low economic value because it can only be extracted naturally from plants at very low concentrations. Large amounts of value-added products can be generated through the metabolic engineering of microbial hosts [8-10]. Unlike plants, microorganisms 
usually do not carry a specific GPP synthase (GPPS) and cannot make monoterpenes with the exception of a few winemaking Saccharomyces cerevisiae strains which manage to do so (5 $\mathrm{mg} / \mathrm{L}$ monoterpenes) [11-13]. In recent years, geraniol has been successfully heterologously produced in Escherichia coli and S. cerevisiae. Mutations in farnesyl diphosphate synthase (FPPS) allow GPP release for monoterpene biosynthesis in recombinant microorganisms harboring monoterpene synthases $[11,14,15]$. A recent study demonstrated that GPP accumulation in yeast bearing mutated FPPS enabled geraniol formation in the absence of a heterologous geraniol synthase probably through endogenous dephosphorylation $[11,14]$. Geraniol can also be generated even in the absence of specific GPPS or mutated FPPS in E. coli by simply overexpressing an Ocimum basilicum geraniol synthase (GES), although the GPP release mechanism remains unclear [16]. By co-overexpression of a FPPS mutant and GES in S. cerevisiae, $5 \mathrm{mg} / \mathrm{L}$ geraniol was obtained after 7 days of culture [11]. Production of geraniol was further increased to $36.04 \mathrm{mg} / \mathrm{L}$ in S. cerevisiae harboring both regulator gene $M A F 1$ and GES after $48 \mathrm{~h}$ of culture by overexpressing key rate-limiting enzymes of the MVA pathway [17]. So far, maximum geraniol $(182.5 \mathrm{mg} / \mathrm{L})$ has been produced by geraniol dehydrogenase mutant $E$. coli with the whole MVA pathway and GES after $48 \mathrm{~h}$ of culture [5]. However, the titer is still too low for industrial applications.

In addition, geraniol usually undergoes biotransformation to other terpenoids in aromatic plants, which influences the quality of distilled essential oils $[18,19]$. The conversion of geraniol to trans-citral in Cymbopogon flexuosus leaves is catalyzed by $\mathrm{NADP}^{+}$-dependent geraniol dehydrogenase [20]. Similarly, some wine yeasts can modify the free terpenoid contents, although they only have limited capability to produce monoterpenoids [21-23]. $S$. cerevisiae is able to convert geraniol into citronellol under the catalysis of enzyme OYE2 [24], and ATF1 alcohol acetyltransferase is involved in the acetylation of geraniol during S. cerevisiae fermentation [24]. Unlike the extensive studies on yeast, the bioconversion of geraniol in $E$. coli has seldom been referred and only until recently, geraniol has been dehydrogenized and isomerized into other geranoids (nerol, neral, and geranial) in E. coli by enzyme YjgB [5].

Thereby motivated, we created an effective geraniolbiosynthesizing strain and developed a new high-performance fermentation strategy to increase geraniol production. In addition, we observed the interconversion between geraniol and geranyl acetate in E. coli. The mechanism by which geranyl acetate was hydrolyzed into geraniol was thus investigated and controlled to further increase geraniol production.

\section{Results and discussion}

\section{Regulated biosynthesis of geraniol from glucose in E. coli}

A highly efficient strain LWG6 was constructed to produce geraniol from glucose in E. coli, comprising a heterotic MVA pathway from Enterococcus faecalis and $S$. cerevisiae, GPP synthase GPPS2 from Abies grandis, and codon-optimized GES from O. basilicum. This biosynthetic pathway (Fig. 1) was adapted from a previous study with genes from different origins [5]. It has previously been reported that GPP was efficiently synthesized by this heterotic MVA pathway and GPP synthase from $A$. grandis $[25,26]$. After $48 \mathrm{~h}$ of shake-flask culture $\left(\mathrm{OD}_{600}=2\right), 68.6 \pm 3 \mathrm{mg} / \mathrm{L}$ geraniol was obtained from glucose by LWG6, while the control strain LWG10 without GES failed to produce geraniol. The geraniol production efficiency of strain LWG6 $\left(34.3 \mathrm{mg} / \mathrm{L} / \mathrm{OD}_{600}\right)$ doubled that of strain GEOLW (about $16 \mathrm{mg} / \mathrm{L} / \mathrm{OD}_{600}$ ) after $48 \mathrm{~h}$ of culture in flask [5], which followed a similar geraniol synthesis pathway to that of wild type E. coli MG 1655. Accordingly, LWG6 was a promising strain for geraniol synthesis.

\section{Geranyl acetate formation in E. coli under fed-batch fermentation condition}

The fed-batch fermentation was carried out with LWG6 based on the results obtained above in shake flask. Geraniol accumulation was monitored over the course of fermentation (Fig. 2). The highest concentration of geraniol was just $78.8 \mathrm{mg} / \mathrm{L}$ after being induced by isopropyl $\beta$-D-thiogalactoside (IPTG) for $5 \mathrm{~h}$. The low titer then plummeted to $12.9 \mathrm{mg} / \mathrm{L}$ after $24 \mathrm{~h}$, which may be ascribed to the volatilization of geraniol during fermentation and the accumulated toxicity may further prevent its synthesis by engineered E. coli $[27,28]$. To prove the volatility of geraniol during fermentation, an authentic geraniol standard was fed to the culture of $E$. coli BL21 (DE3) in a $5 \mathrm{~L}$ fermentor. As shown in Fig. 3a, $81.4 \%$ of fed geraniol is lost during the first $5 \mathrm{~h}$ of fermentation, probably through volatilization. In order to prevent volatilization, isopropyl myristate that also can reduce monoterpene toxicity was added [27], forming an aqueous-organic two-phase culture system. With this system, the amount of fed geraniol was kept stable during $20 \mathrm{~h}$ of culture (Fig. 3b), which was conducive to geraniol fermentation.

Moreover, the dehydrogenation and isomerization of geraniol into other geranoids (nerol, neral, and geranial) may also be responsible for the loss of geraniol [5]. Only $10.8 \mathrm{mg} / \mathrm{L}$ nerol was detected at $18 \mathrm{~h}$, which descended to zero at the end of fermentation (Fig. 2). Instead of nerol, neral, and geranial, a new compound appeared at $18 \mathrm{~h}$ of culture, which was identified as geranyl acetate 


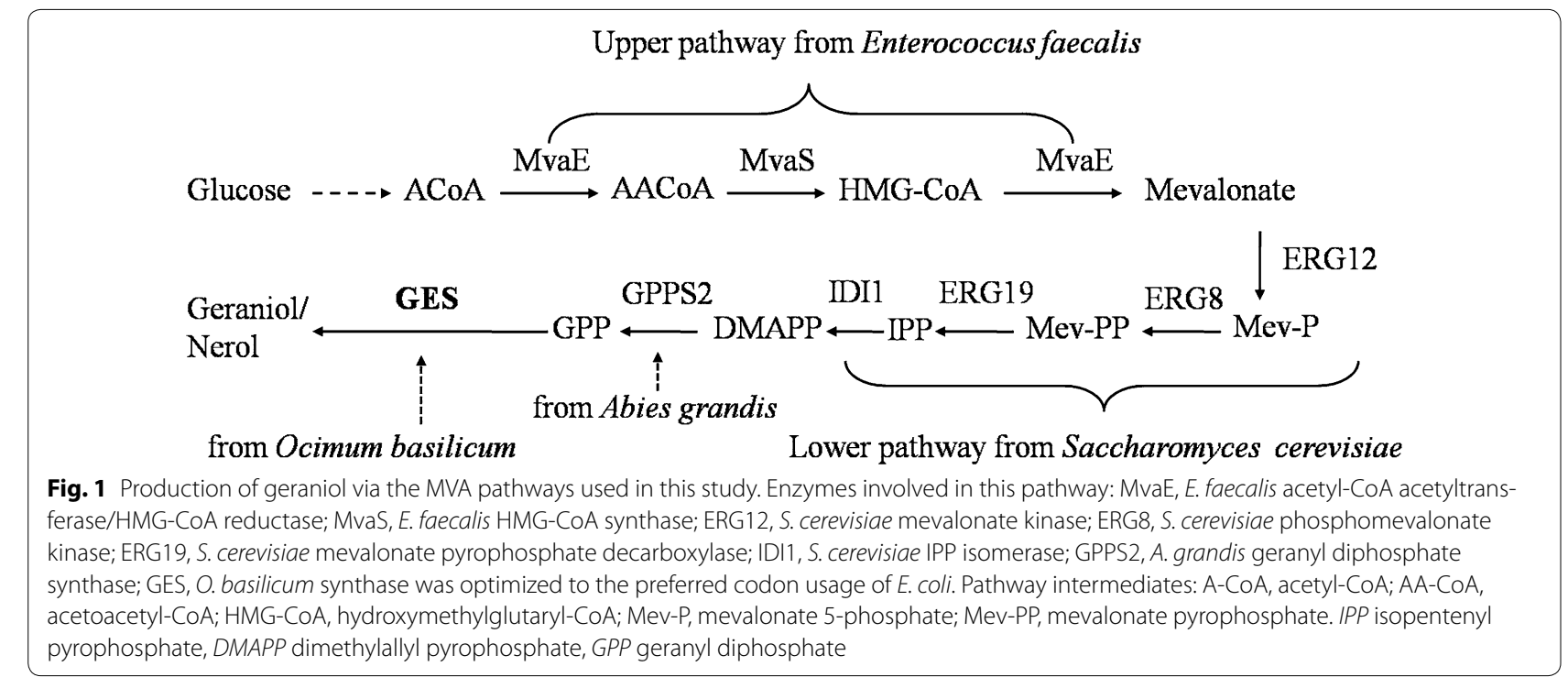

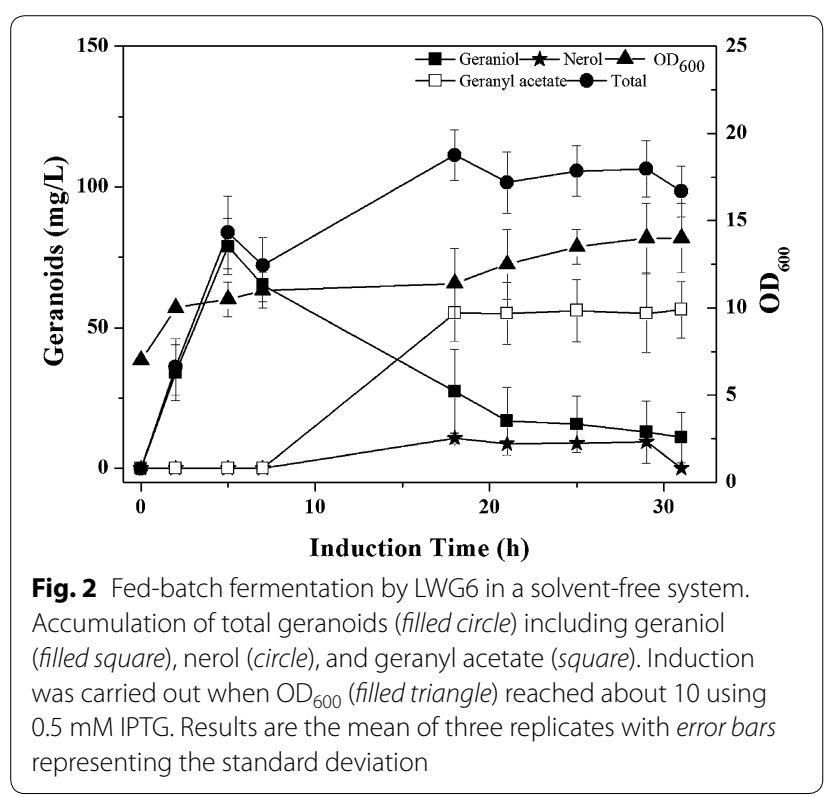

by GC-MS analysis. At the end of fermentation, geranyl acetate accounted for $83.7 \%$ of total geranoids.

The formation of geranyl acetate was analyzed. Firstly, cat gene in plasmid pSTV28 encoding chloramphenicol acetyltransferase (CAT) is known to be responsible for chloramphenicol resistance, which exhibits non-specific esterification activity toward esterification of geraniol into geranyl acetate [5]. Plasmid pACYDuet-1 used in our study also harbors CAT, forming geranyl acetate. Secondly, in a previous study, acetyltransferase ATF1 (EC 2.3.1.84) from S. cerevisiae mainly contributed to geranyl acetate synthesis [24]. It is highly possible that similar functional enzymes of O-acetyltransferase (EC 2.3.1.9) also exist in E. coli and cause the esterification of geraniol therein [29]. This postulation was supported by the fed experiment shown in Fig. 3b. Geraniol decreased after $20 \mathrm{~h}$ of culture along with the accumulation of geranyl acetate. After $40 \mathrm{~h}$ of culture, more than $40 \%$ of fed geraniol was converted into geranyl acetate $(128.3 \mathrm{mg} / \mathrm{L})$ by $E$. coli BL21 (DE3). Nerol was also detected but the total production was lower than $8 \%$, suggesting that geraniol was not lost mainly through dehydrogenation in E. coli BL21 (DE3) under such fed-batch conditions. Geranial and neral were not found and the amounts of total geranoids (geraniol, nerol, and geranyl acetate) were relatively stable during fermentation.

\section{Conversion of geranyl acetate to geraniol by AES from $E$. coli}

Simple gene knockout may not effectively prevent geranyl acetate synthesis, so geraniol production can feasibly be augmented by converting geranyl acetate to geraniol in engineered E. coli. Acetylesterase GAE (EC 3.1.1.6) from Cymbopogon martinii is involved in the transformation of geranyl acetate into geraniol [18]. Acetylesterase (Aes, EC 3.1.1.6) also exists in E. coli, although its effect on geranyl acetate is still not clear [30]. In our study, Aes was overexpressed in E. coli BL21 (DE3), the activity of which was indicated by incubating geranyl acetate under defined conditions and monitoring the amount of produced geraniol with GC-MS. Since about $75 \%$ of geranyl acetate was converted into geraniol after $2 \mathrm{~h}$ of incubation, E. coli was capable of geranyl acetate hydrolyzation. No geraniol was produced in the control experiment using boiled and denatured enzyme. 

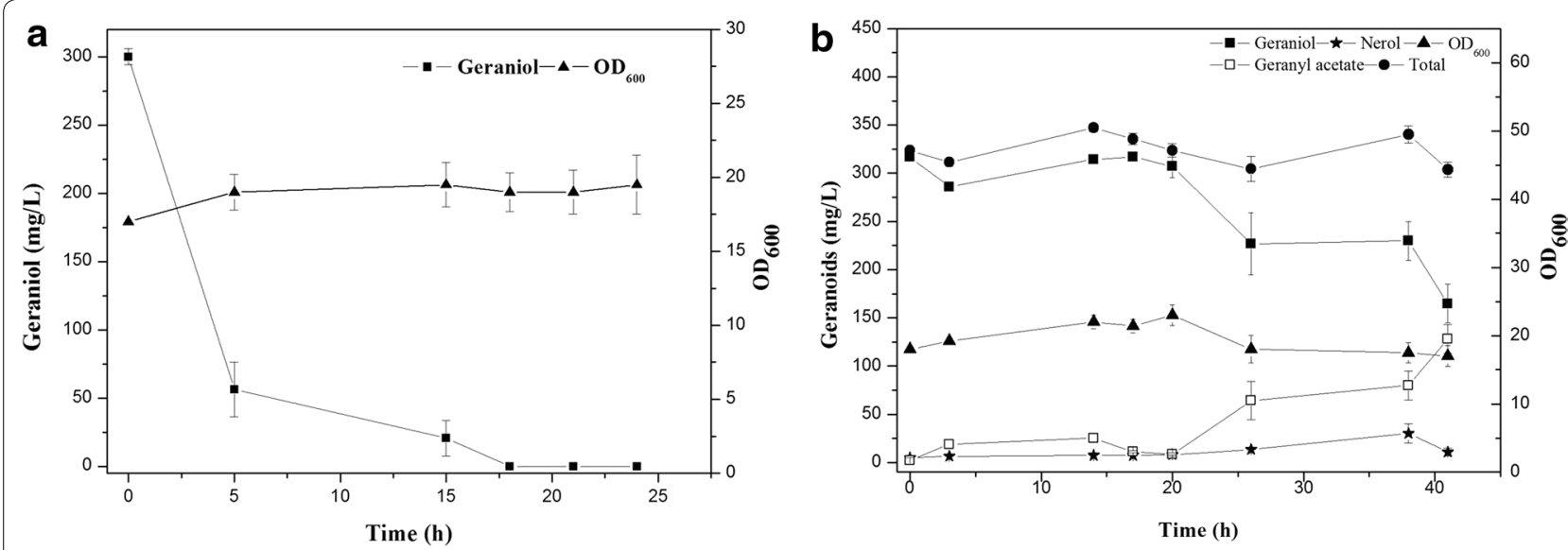

Fig. 3 Geranyl acetate feeding experiments by E. coli BL21 (DE3). a Feeding experiments in a solvent-free system. b Feeding experiments in aqueous-organic two-phase culture system. Isopropyl myristate was added when $\mathrm{OD}_{600}$ (filled triangle) reached about 20 according to one over ten of the fermentation medium volume. Accumulation of total geranoids (filled circle) including geraniol (filled square), nerol (circle), and geranyl acetate (square). Results are the mean of three replicates with error bars representing the standard deviation

\section{Geraniol production under fed-batch fermentation} with biotransformation of geranyl acetate to geraniol Considering the effect of Aes, geraniol production can feasibly be increased through biotransformation of geranyl acetate to geraniol during fermentation. It can be allowed by overexpression of Aes in LWG6 which, however, further burdens the cell metabolism system, since eight heterologous genes have already been designed to be overexpressed. Moreover, the above transformation can be realized by regulating fermentation. In the absence of glucose, E. coli cells reuse acetate [31], thereby facilitating the formation of geraniol catalyzed by Aes. Geraniol feeding experiment was used to identify whether geranyl acetate, which was esterified from geraniol, can reproduce geraniol by this glucose starvation strategy. The control strain LWG10 was used in feeding experiment to ensure similar conversion of geraniol to geranyl acetate. As shown in Fig. 4a, fed geraniol is lost quickly by LWG10 and about $86 \%$ of geraniol is converted to geranyl acetate at $21 \mathrm{~h}$. From 21 to $28 \mathrm{~h}$, the geraniol amount remained stable, suggesting the reaction between geraniol and geranyl acetate reached equilibrium. Then glucose supply was stopped at $28 \mathrm{~h}$, and geraniol production rose from 33 to $160 \mathrm{mg} / \mathrm{L}$ at $39 \mathrm{~h}$ when residual glucose was exhausted, probably because the reuse of acetate disturbed the reaction balance and moved the reaction toward geraniol formation under the catalysis of Aes. For comparison, glucose was continuously added and most of geraniol was quickly converted to geranyl acetate that was kept at a low concentration thereafter (Fig. 4b).

Thus, a new fermentation strategy was established based on the above results. First, isopropyl myristate was added to form an aqueous-organic two-phase culture system. Second, glucose starvation was employed to convert geranyl acetate into geraniol. Therefore, this new fermentation condition was used with LWG6 (Fig. 5). At the beginning, both geraniol and geranyl acetate increased quickly and $\mathrm{OD}_{600}$ value of the bacterial culture increased from 20 to 32 rapidly. The titers of geraniol and geranyl acetate reached 1.04 and $1.01 \mathrm{~g} / \mathrm{L}$ at $24 \mathrm{~h}$ of culture, respectively. During the next $24 \mathrm{~h}$, geranyl acetate increased to $1.43 \mathrm{~g} / \mathrm{L}$ with decreasing geraniol, suggesting that the strain no longer synthesized geraniol that was converted to geranyl acetate. Then, glucose supply was stopped at $48 \mathrm{~h}$ and the culture was continued under the glucose starvation condition. As expected, geranyl acetate was converted to geraniol after $56 \mathrm{~h}$ of culture while $\mathrm{OD}_{600}$ value of the bacterial culture began to decline slightly. At the end of fermentation, the concentration of geraniol reached maximum $(2.0 \mathrm{~g} / \mathrm{L})$, and the yield (from glucose to geraniol) was $14 \%$ which is approximately 11 -fold that reported before [5]. Geranyl acetate production was reduced to $0.16 \mathrm{~g} / \mathrm{L}$ at $68 \mathrm{~h}$. Geraniol or neral was not detected while nerol was kept at a very low concentration during fermentation $(0.05 \mathrm{~g} / \mathrm{L}$ at $52 \mathrm{~h})$. About $1.27 \mathrm{~g} / \mathrm{L}(88.8 \%)$ geranyl acetate was successfully converted to geraniol during the later stage of fermentation by glucose starvation.

Although higher concentration of geraniol was obtained in this study, geraniol production needs to be elevated at least 3 to 4 times in the future to meet the requirements of industrialization. Of all possible improvement strategies, engineering of the host can be realized by employing a chromosome integration technique to decrease the cell growth burden that results from overexpression of heterologous genes. Another 

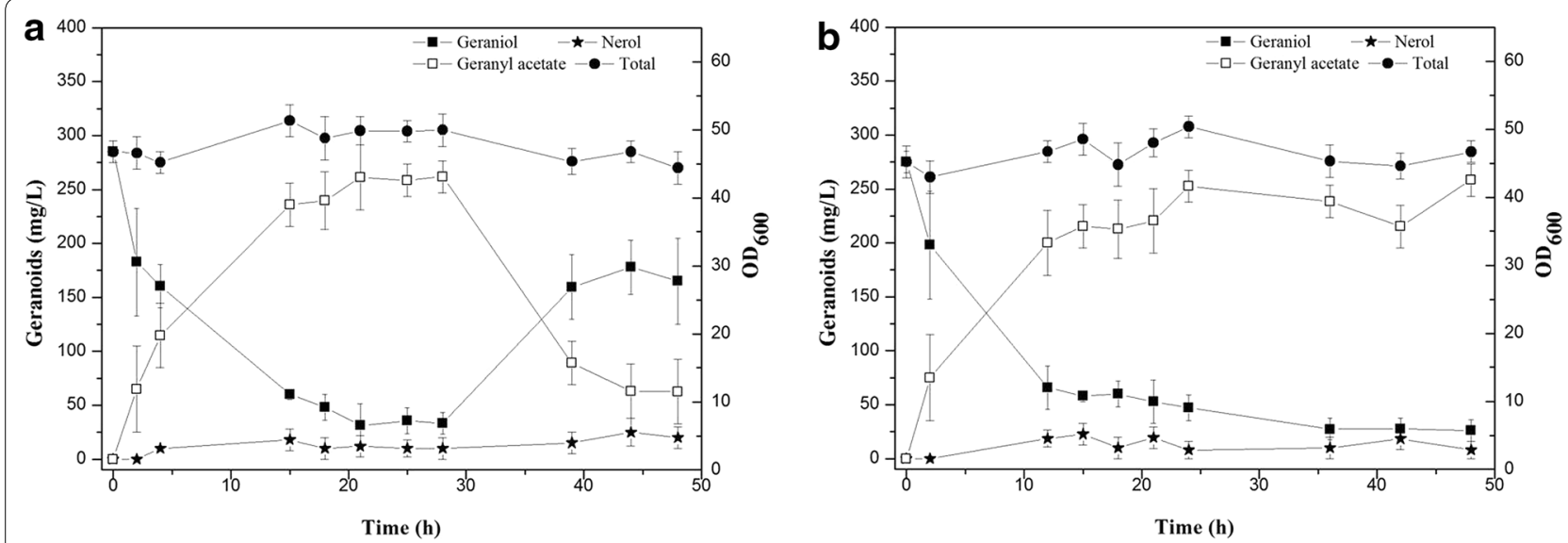

Fig. 4 Geranyl acetate feeding experiments by LWG10. a Glucose addition was stopped after $28 \mathrm{~h}$ of culture. b The residual glucose was maintained below $5 \mathrm{~g} / \mathrm{L}$ by a feeding solution containing $800 \mathrm{~g} / \mathrm{L}$ glucose at appropriate rates. Isopropyl myristate was added when $\mathrm{OD}_{600}$ reached about 20 according to one over ten of the fermentation medium volume. Accumulation of total geranoids (filled circle) including geraniol (filled square), nerol (circle), and geranyl acetate (square). Results are the mean of three replicates with error bars representing the standard deviation

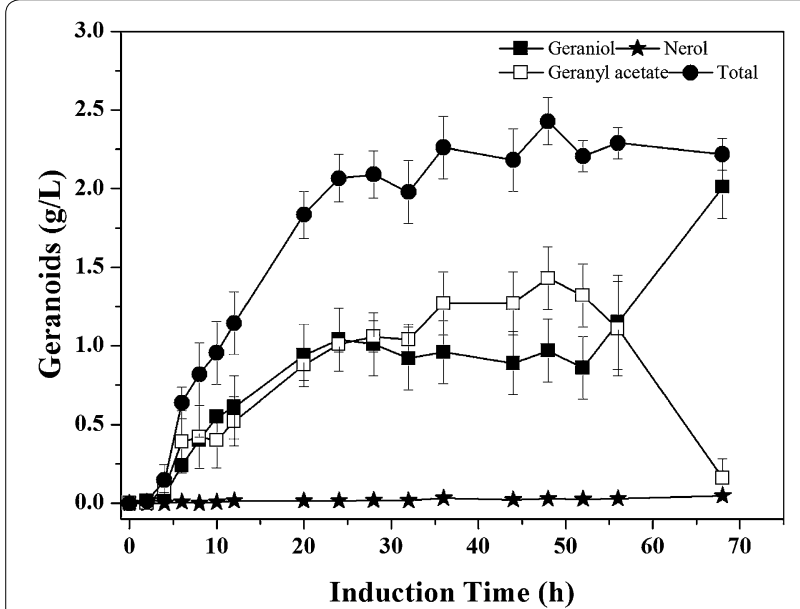

Fig. 5 Geraniol production under fed-batch fermentation with biotransformation of geranyl acetate to geraniol. Accumulation of total geranoids (filled circle) including geraniol (filled square), nerol (circle), and geranyl acetate (square). Induction was carried out when $\mathrm{OD}_{600}$ reached about 20 using 0.5 mM IPTG. Isopropyl myristate was added $4 \mathrm{~h}$ after IPTG induction according to one over ten of the fermentation medium volume, and glucose addition was stopped after $48 \mathrm{~h}$ of culture. Results are the mean of three replicates with error bars representing the standard deviation

approach is optimization of fermentation conditions such as induction time, inoculum age, and organic solvent for geraniol production.

\section{Conclusions}

In summary, an efficient strain LWG6 was constructed and an isopropyl myristate-overlaid two-phase fed-batch fermentation system was established to biosynthesize geraniol from glucose. For the first time, the interconversion between geraniol and geranyl acetate by $E$. coli was observed, and Aes from E. coli was involved in the hydrolysis of geranyl acetate. Geraniol production reached up to $2.0 \mathrm{~g} / \mathrm{L}$ with biotransformation of $88.8 \%$ geranyl acetate to geraniol under controlled fermentation condition, which is the highest from glucose hitherto. This study provided a new strategy for geraniol biosynthesis.

\section{Methods}

\section{Medium and culture conditions}

Luria broth (LB) medium (10 g/L tryptone, $10 \mathrm{~g} / \mathrm{L} \mathrm{NaCl}$, and $5 \mathrm{~g} / \mathrm{L}$ yeast extract) was used for gene cloning and shake-flask fermentation. For geraniol production, recombinant strains were cultured in shake-flask or fedbatch fermentation with the medium containing $20 \mathrm{~g} / \mathrm{L}$ glucose, $9.8 \mathrm{~g} / \mathrm{L} \mathrm{K}_{2} \mathrm{HPO}_{4}, 5 \mathrm{~g} / \mathrm{L}$ beef extract, $0.3 \mathrm{~g} / \mathrm{L}$ ferric ammonium citrate, $2.1 \mathrm{~g} / \mathrm{L}$ citric acid monohydrate, and $0.06 \mathrm{~g} / \mathrm{L} \mathrm{MgSO}_{4}$ as well as $1 \mathrm{ml}$ of trace element solution which included $0.37 \mathrm{~g} / \mathrm{L}\left(\mathrm{NH}_{4}\right)_{6} \mathrm{Mo}_{7} \mathrm{O}_{24} \cdot 4 \mathrm{H}_{2} \mathrm{O}, 0.29 \mathrm{~g} / \mathrm{L}$ $\mathrm{ZnSO}_{4} \cdot 7 \mathrm{H}_{2} \mathrm{O}, 2.47 \mathrm{~g} / \mathrm{L} \mathrm{H}_{3} \mathrm{BO}_{4}, 0.25 \mathrm{~g} / \mathrm{L} \mathrm{CuSO} \cdot 5 \mathrm{H}_{2} \mathrm{O}$, and $1.58 \mathrm{~g} / \mathrm{L} \mathrm{MnCl}_{2} \cdot 4 \mathrm{H}_{2} \mathrm{O}$. Appropriate antibiotics were added to the culture medium according to selectable marker gene of each plasmid listed in Table 1 at the following concentrations: ampicillin (Amp, $100 \mathrm{mg} / \mathrm{ml}$ ), kanamycin (Kan, $50 \mathrm{mg} / \mathrm{ml}$ ), and chloramphenicol $(\mathrm{Cm}$, $34 \mathrm{mg} / \mathrm{ml})$.

\section{Strains and plasmids}

All strains and plasmids used in this study are listed in Table 1. The nucleotide sequences of GES gene from O. basilicum (sweet basil) (GenBank No. AY362553.1) were analyzed (http://www.genscript.com/cgi-bin/tools/ 
Table 1 Strains and plasmids used in this study

\begin{tabular}{|c|c|c|}
\hline Name & Relevant characteristics & References \\
\hline \multicolumn{3}{|l|}{ primers $^{\mathrm{a}}$} \\
\hline GES-rbs-F & GGAAGATCTAGGAGGTAAAAAATATGTCTTGCGCTCGTATCACCG & This study \\
\hline GES-R & CCGCTCGAGTTACTGGGTGAAGAACAGAGCG & This study \\
\hline Aes-F-Ncol & CCCATGGCTATGAAGCCGGAAAACAAACT & This study \\
\hline Aes-R-EcoRl & GGAATTCCTAAAGCTGAGCGGTAAAGAACTG & This study \\
\hline \multicolumn{3}{|l|}{ Strains } \\
\hline BL21(DE3) & 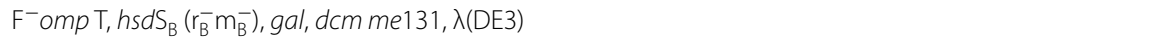 & Invitrogen \\
\hline LWG6 & E.coli BL21(DE3)/pLWG6, pYJM14 & This study \\
\hline LWG10 & E.coli BL21(DE3)/p YJM26, pYJM14 & This study \\
\hline LWG11 & E.coli BL21(DE3)/p LWG11 & This study \\
\hline \multicolumn{3}{|l|}{ Plasmids } \\
\hline pET-30a & F1 (pBR322), Kan & Novagen \\
\hline $\mathrm{pGH} / \mathrm{GES}$ & pGH carrying GES from O. basilicum & This study \\
\hline pLWG 6 & pACYCDuet-1 carrying mvaE and mvaS from E. faecalis, GPPS2 from A. grandis,GES from O. basilicum, Cm & This study \\
\hline pLWG11 & pET-30a carrying aes from E. coli, Kan & This study \\
\hline pYJM26 & pACYCDuet-1 carrying mvaE and mvaS from E. faecalis, GPPS2 from A. grandis, Cm & {$[27]$} \\
\hline pYJM14 & pTrcHis2B carrying ERG12, ERG8, ERG19 and IDI1 from S.cerevisiae, Amp & {$[27]$} \\
\hline
\end{tabular}

a Restriction sites are underlined

rare_codon_analysis) and optimized to the preferred codon of E. coli (http://www.jcat.de/) online. The codonoptimized GES gene was synthesized by Genray Company with plasmid pGH as vector pGH/GES. GES gene was PCR-amplified from plasmid DNA of pGH/GES with primer set GES-rbs-F/GES-R. The PCR products were digested with $B g l \mathrm{II} / \mathrm{XhoI}$, respectively, and introduced into the corresponding sites of pYJM26 to create pLWG 6. Plasmids pLWG 6 and pYJM14 were co-expressed in E. coli BL21 (DE3) to form strain LWG6. Aes was PCRamplified from genomic DNA of BL21 (DE3) with primer set Aes-F-NcoI/Aes-R-EcoRI. The PCR product digested with $N c o$ I and EcoRI was cloned into pET30a cut with the same restriction enzymes, creating pLWG11.

\section{Enzyme extraction and assay}

LWG11 was cultured in LB broth and induced by IPTG at a final concentration of $0.1 \mathrm{mM}$ when $\mathrm{OD}_{600}$ of the bacterial culture reached 0.6-0.8. After being incubated at $30{ }^{\circ} \mathrm{C}$ for $4-6 \mathrm{~h}$, the cells were harvested by centrifugation at $6000 \mathrm{~g}$ for $5 \mathrm{~min}$, washed with distilled water, and then resuspended in $0.5 \mathrm{M}$ Tris- $\mathrm{HCl}(\mathrm{pH} 8)$. All the extraction procedures were carried out at $4{ }^{\circ} \mathrm{C}$. The cells were broken by sonic treatment for $10 \mathrm{~min}$ at $0{ }^{\circ} \mathrm{C}(3 \mathrm{~s}$ pulse on, 3 s pulse off, $40 \mathrm{~W}$, Sonics VCX130, China). The Aes activity was determined by GC-MS monitoring of geraniol produced by the hydrolysis of geranyl acetate. The assay system consisted of $0.05 \mathrm{M}$ Tris- $\mathrm{Cl}, \mathrm{pH}$ 8.0, $5 \mathrm{mM} \mathrm{MgSO}_{4}, 1 \mathrm{mM}$ DTE, $2 \mathrm{mM}$ geranyl acetate, and the enzyme extract (about $0.4 \mathrm{mg}$ protein) in a total volume of $0.5 \mathrm{ml}$. The reaction mixture was incubated at $30{ }^{\circ} \mathrm{C}$ in a sealed capped tube for $2 \mathrm{~h}$. A blank control with boiled enzyme was also run simultaneously.

\section{Shake-flask cultures}

A single colony of LWG6 was grown in LB broth overnight at $37{ }^{\circ} \mathrm{C}$. The culture was used to inoculate the same medium (1:100 dilution) and grown at $37{ }^{\circ} \mathrm{C}$ until an $\mathrm{OD}_{600}$ of $0.6-0.8$ was reached. IPTG was added to a final concentration of $0.1 \mathrm{mM}$, and the culture was further incubated at $30{ }^{\circ} \mathrm{C}$ for $48 \mathrm{~h}$. The samples were added with the same volume of ethyl acetate, vortexed briefly, and centrifuged to separate the phases, and the organic phase was analyzed by GC-MS. LWG10 strain was used as control. The experiment was performed in triplicate.

\section{Fed-batch fermentation for geraniol biosynthesis from glucose}

LWG6 strain was grown overnight at $37^{\circ} \mathrm{C}$ in $100 \mathrm{ml}$ of LB medium and inoculated to a 5-L fermentor (BIOSTAT B plus MO5L, Sartorius, Germany) containing $2 \mathrm{~L}$ of fermentation medium. The temperature was maintained at $37{ }^{\circ} \mathrm{C}$, and $\mathrm{pH}$ was maintained at 7.0 by automatically adding ammonia. Antifoam 204 was used to prohibit foam development. The stirring speed was first set at $400 \mathrm{rpm}$ to maintain a $20 \%$ saturation of dissolved oxygen. The expression of plasmid-born exogenous gene(s) for geraniol production was induced with $0.5 \mathrm{mM}$ IPTG at $30{ }^{\circ} \mathrm{C}$. During the course of fermentation, residual glucose was measured using a glucose analyzer (SBA-40D, 
China) and maintained below $5 \mathrm{~g} / \mathrm{L}$ by a feeding solution containing $800 \mathrm{~g} / \mathrm{L}$ glucose at appropriate rates. The samples were added with the same volume of ethyl acetate, vortexed briefly, and centrifuged to separate the phases, and the organic phase was analyzed.

To prevent violation, isopropyl myristate was added $4 \mathrm{~h}$ after IPTG induction according to one over ten of the fermentation medium volume. Glucose was stopped feeding after $48 \mathrm{~h}$ of culture. The samples were collected on time, and the organic phase was separated by centrifugation at $13,000 \mathrm{rpm}$ for $10 \mathrm{~min}$, then added with ten volumes of ethyl acetate and analyzed by GC-MS.

\section{Geranyl acetate feeding experiments}

About $300 \mathrm{mg}$ per liter medium of geraniol was fed to the cultures of E. coli BL21 (DE3) or LWG10 $\left(\mathrm{OD}_{600}\right.$ at 20) to investigate the fate of geraniol during fermentation. Other fermentation conditions were the same as above.

\section{Geraniol characterization by GC-MS}

Putative geraniol products were identified by GC-MS. A HP-INNOWAX capillary column $(30 \mathrm{~m} \times 0.25 \mathrm{~mm}$; $0.25-\mu \mathrm{m}$-film thickness; Agilent Technologies) was used. The oven temperature was initially held at $80^{\circ} \mathrm{C}$ for $1 \mathrm{~min}$ and sequentially increased at the rate of $10^{\circ} \mathrm{C} / \mathrm{min}$ to 180 and $30{ }^{\circ} \mathrm{C} / \mathrm{min}$ to $250^{\circ} \mathrm{C}$. Peak identification was based on a relative retention time and total ion mass spectral comparison with an external standard (Sigma-Aldrich, USA). The peak areas were converted into concentrations in comparison with standard curves plotted with a set of known concentrations of standards.

\footnotetext{
Abbreviations

Aes: acetylesterase; MVA: mevalonate; GPP: geranyl diphosphate; GPPS: geranyl diphosphate synthase; FPPS: farnesyl diphosphate synthase; GES: Ocimum basilicum geraniol synthase; Amp: ampicillin; Kan: kanamycin; Cm: chloramphenicol; IPTG: isopropyl $\beta$-D-thiogalactoside.

\begin{abstract}
Authors' contributions
$H L$ and $W L$ developed the idea for the study. WL and MX designed the research, did the literature review, and prepared the manuscript. YC and JG helped to revise the manuscript. XX, RZ, TC, and XXL did the lab work, detection. WL and YC drafted the initial manuscript together and all authors contributed in analyzing the data and writing the draft. All authors read and approved the final manuscript.
\end{abstract} plasmid construction, strain cultivation, fed-batch fermentation, and product
}

\section{Author details \\ ${ }^{1}$ CAS Key Laboratory of Bio-Based Materials, Qingdao Institute of Bioenergy and Bioprocess Technology, Chinese Academy of Sciences, Qingdao 266101, China. ${ }^{2}$ Key Laboratory of Green Process and Engineering, Institute of Process Engineering, Chinese Academy of Sciences, Beijing 10090, China. ${ }^{3}$ Univer- sity of Chinese Academy of Sciences, Beijing 100049, China. ${ }^{4}$ Department of Chemistry, University of Nebraska-Lincoln, Lincoln, NE 68588, USA.}

\section{Acknowledgements}

This work was financially supported by the National Natural Science Foundation of China (21376255), Key Program of the Chinese Academy of Sciences
(KGZD-EW-606-1-3), and Taishan Scholars Climbing Program of Shandong (tspd20150210).

\section{Competing interests}

The authors declare that they have no competing interests.

Received: 29 October 2015 Accepted: 19 February 2016

Published online: 11 March 2016

\section{References}

1. Chen W, Viljoen AM. Geraniol-a review of a commercially important fragrance material. S Afr J Bot. 2010;76(4):643-51.

2. Lapczynski A, Bhatia SP, Foxenberg RJ, Letizia CS, Api AM. Fragrance material review on geraniol. Food Chem Toxicol. 2008;46(Suppl 11):S160-70.

3. Polo M, Crespo R. Bravo Md. Geraniol and simvastatin show a synergistic effect on a human hepatocarcinoma cell line. Cell Biochem Funct. 2011;29(6):452-8

4. Peralta-Yahya PP, Keasling JD. Advanced biofuel production in microbes. Biotechnol J. 2010;5(2):147-62.

5. Zhou J, Wang C, Yoon S-H, Jang H-J, Choi E-S, Kim S-W. Engineering Escherichia coli for selective geraniol production with minimized endogenous dehydrogenation. J Biotechnol. 2014;169:42-50.

6. Goldstein JL, Brown MS. Regulation of the mevalonate pathway. Nature. 1990:343(6257):425-30.

7. Rohmer M. The discovery of a mevalonate-independent pathway for isoprenoid biosynthesis in bacteria, algae and higher plants. Nat Prod Rep. 1999;16(5):565-74

8. Chang MC, Keasling JD. Production of isoprenoid pharmaceuticals by engineered microbes. Nat Chem Biol. 2006;2(12):674-81.

9. Clomburg JM, Gonzalez R. Biofuel production in Escherichia coli: the role of metabolic engineering and synthetic biology. Appl Microbiol Biotechnol. 2010;86(2):419-34.

10. Leonard E, Lim K-H, Saw P-N, Koffas MA. Engineering central metabolic pathways for high-level flavonoid production in Escherichia coli. Appl Environ Microbiol. 2007:73(12):3877-86.

11. Fischer MJ, Meyer S, Claudel P, Bergdoll M, Karst F. Metabolic engineering of monoterpene synthesis in yeast. Biotechnol Bioeng. 2011;108(8):1883-92.

12. Carrau FM, Medina K, Boido E, Farina L, Gaggero C, Dellacassa E, et al. De novo synthesis of monoterpenes by Saccharomyces cerevisiae wine yeasts. FEMS Microbiol Lett. 2005;243(1):107-15.

13. Zea L, Moreno J, Ortega JM, Medina M. Content of free terpenic compounds in cells and musts during vinification with three Saccharomyces cerevisiae races. J Agric Food Chem. 1995;43(4):1110-4.

14. Oswald M, Fischer M, Dirninger N, Karst F. Monoterpenoid biosynthesis in Saccharomyces cerevisiae. FEMS Yeast Res. 2007;7(3):413-21.

15. Blanchard L, Karst F. Characterization of a lysine-to-glutamic acid mutation in a conservative sequence of farnesyl diphosphate synthase from Saccharomyces cerevisiae. Gene. 1993;125(2):185-9.

16. Fischer MJ, Meyer S, Claudel P, Perrin M, Ginglinger JF, Gertz C, et al. Specificity of Ocimum basilicum geraniol synthase modified by its expression in different heterologous systems. J Biotechnol. 2013;163(1):24-9.

17. Liu J, Zhang W, Du G, Chen J, Zhou J. Over production of geraniol by enhanced precursor supply in Saccharomyces cerevisiae. J Biotechnol. 2013;168(4):446-51.

18. Dubey VS, Luthra R. Biotransformation of geranyl acetate to geraniol during palmarosa (Cymbopogon martinii, Roxb. wats. var. motia) inflorescence development. Phytochemistry. 2001;57(5):675-80.

19. Cormier F, Ambid C. Extractive bioconversion of geraniol by a Vitis vinifera cell suspension employing a two-phase system. Plant Cell Rep. 1987;6(6):427-30

20. Sangwan RS, Singh-Sangwan N, Luthra R. Metabolism of acyclic monoterpenes: partial purification and properties of geraniol dehydrogenase from lemongrass (Cymbopogon flexuosus Stapf.) leaves. J Plant Physiol. 1993;142(2):129-34

21. Fia G, Giovani G, Rosi I. Study of $\beta$-glucosidase production by wine-related yeasts during alcoholic fermentation. A new rapid 
fluorimetric method to determine enzymatic activity. J Appl Microbiol. 2005;99(3):509-17.

22. Arifin $A A$, Don MM, Uzir MH. Baker's yeast mediated biotransformation of geraniol into citronellol using a continuous-closed-gas-loop bioreactor (CCGLB) system. Biochem Eng J. 2011;56(3):219-24.

23. Gamero A, Manzanares P, Querol A, Belloch C. Monoterpene alcohols release and bioconversion by Saccharomyces species and hybrids. Int $J$ Food Microbiol. 2011;145(1):92-7.

24. Steyer D, Erny C, Claudel P, Riveill G, Karst F, Legras J-L. Genetic analysis of geraniol metabolism during fermentation. Food Microbiol. 2013;33(2):228-34.

25. Yang J, Xian M, Su S, Zhao G, Nie Q, Jiang XL, Zheng YN, Liu W. Enhancing production of bio-Isoprene using hybrid MVA pathway and isoprene synthase in E. coli. PLoS One. 2012;7(4):e33509.

26. Yang J, Nie Q, Ren M, Feng H, Jiang X, Zheng Y, et al. Metabolic engineering of Escherichia coli for the biosynthesis of alpha-pinene. Biotechnol Biofuels. 2013;6(1):60.

27. Brennan TC, Turner CD, Krömer JO, Nielsen LK. Alleviating monoterpene toxicity using a two-phase extractive fermentation for the bioproduction of jet fuel mixtures in Saccharomyces cerevisiae. Biotechnol Bioeng. 2012;109(10):2513-22.
28. Wang C, Yoon SH, Shah AA, Chung YR, Kim JY, Choi ES, et al. Farnesol production from Escherichia coli by harnessing the exogenous mevalonate pathway. Biotechnol Bioeng. 2010;107(3):421-9.

29. Kursula P, Sikkilä H, Fukao T, Kondo N, Wierenga RK. High resolution crystal structures of human cytosolic thiolase (CT): a comparison of the active sites of human CT, bacterial thiolase, and bacterial KAS I. J Mol Biol. 2005;347(1):189-201.

30. Farias T, Mandrich L, Rossi M, Manco G. Biochemical and thermostability features of acetyl esterase aes from Escherichia coli. Protein Pept Lett. 2007;14(2):165-9.

31. Prieto MA, Galán B, Torres B, Ferrández A, Fernández C, Miñambres B, et al. Aromatic metabolism versus carbon availability: the regulatory network that controls catabolism of less-preferred carbon sources in Escherichia coli. FEMS Microbiol Rev. 2004;28:503-18.

\section{Submit your next manuscript to BioMed Central and we will help you at every step:}

- We accept pre-submission inquiries

- Our selector tool helps you to find the most relevant journal

- We provide round the clock customer support

- Convenient online submission

- Thorough peer review

- Inclusion in PubMed and all major indexing services

- Maximum visibility for your research

Submit your manuscript at www.biomedcentral.com/submit

() Biomed Central 